\section{INSTRUMENTOS CIENTÍFICOS ANTIGUOS EN EL INSTITUTO SAN ISIDRO. RECUPERACIÓN Y CONTEXTUALIZACIÓN}

\author{
Leonor González de la Lastra \\ I.E.S. San Isidro'. C/ Rosario, 19, 28005 Madrid \\ leonor.gonzalez@educa.madrid.org
}

\section{OLD SCIENTIFIC INSTRUMENTS AT THE SAN ISIDRO HIGH SCHOOL. RECOVERY AND CONTEXTUALIZATION.}

\begin{abstract}
This paper presents the results of the labours undertaken in the Instituto San Isidro regarding the conservation, documentation and contextualization of the XIXth century scientific instruments that still belong to this institution. In order to achieve an historical understanding of the collection, the attention is focused on the political and educational decisions taken by the Spanish authorities in the period considered. Besides, particular attention is paid to technological pieces, which in general were used, as in other countries, to illustrate the "applied side" of physics.
\end{abstract}

KEY WORDS: Scientific instrument; technological instrument; physics teaching; technology teaching; Reales Estudios de San Isidro; documentation; instrument maker; XIXth-century; history of Spanish education.

En las líneas que siguen presentamos los resultados de las tareas llevadas a cabo en el instituto San Isidro con objeto de recuperar el material científico antiguo ${ }^{2}$ que esta institución aún conserva, después de que en 1985 se trasladase una parte importante al Museo Nacional de Ciencia y Tecnología (en adelante MUNCYT), y que estuvo destinado a la enseñanza de la Física y la Química durante los siglos XIX y XX.

Comenzaremos con una contextualización de la colección, en la que describiremos someramente los momentos históricos más importantes de la institución y aquéllos en los que tuvo lugar la adquisición de material científico y tecnológico, para ello haremos las pertinentes referencias a las asignaturas y textos que justificaban la necesidad y existencia de este tipo de instrumental, así como a las
RESUMEN: En el presente artículo se tratan las tareas de recuperación y documentación del material científico antiguo existente en el actual instituto San Isidro de Madrid tras el traslado en 1985 de una parte importante al Museo Nacional de Ciencia y Tecnología. Para contextualizar dicha colección, destinada en su mayoría a la enseñanza de la Física en el s. XIX, se estudia su creación y desarrollo, relacionándolos con la historia del centro, así como con los acontecimientos políticos y educativos que condicionaron sus caracteristicas. Asimismo, se señala la presencia en esta colección de instrumentos tecnológicos, tema insuficientemente tratado en los trabajos disponibles, vinculados a las enseñanzas de Física. A la hora de describir las tareas destinadas a recuperar los instrumentos científicos mencionados se describe tanto la metodología seguida como los resultados, ejemplificados en la documentación de dos piezas representativas, una de Física y otra de carácter tecnológico.

PALABRAS CLAVE: Instrumento científico; instrumento tecnológico; enseñanza de la Física; enseñanza de la Tecnología; Reales Estudios de San Isidro; catalogación; fabricante de instrumentos; siglo XIX; historia de la educación española.

cuestiones politicas y económicas que hacian posible su presencia.

En la segunda parte de esta exposición trataremos los trabajos de estudio y recuperación de dicho material; ilustraremos con ejemplos dicha descripción.

1. APUNTES SOBRE LA HISTORIA DE LA COLECCIÓN DE MATERIAL CIENTÍFICO-TECNOLÓGICO DEL INSTITUTO SAN ISIDRO dE MAdRID

Los instrumentos científicos, presentes hoy en día en todos los centros de enseñanza secundaria, han estado asociados al que hoy conocemos como instituto San Isidro desde su 
fundación en el siglo XVII. Los orígenes de este instituto, el más antiguo de la capital, se remontan al denominado Colegio Imperial, centro de estudios superiores a cargo de la Compañía de Jesús desde 1625. Ya en esa época, los jesuitas empleaban instrumentos científicos y técnicos en sus enseñanzas. Es más, alguno de ellos pertenece a las últimas décadas de la centuria anterior a la fundación del Colegio, por lo que, aunque su origen no se ha podido determinar con exactitud, podría relacionarse con la Academia de Matemáticas ${ }^{3}$ o el gabinete del Monasterio de El Escorial.

El hecho de que este tipo de utillaje científico y tecnológico se haya mantenido asociado de forma continuada a la institución mencionada, que además conserva su ubicación y edificio en la calle de Toledo, convierte al conjunto de instrumentos conservado, que representa una parte importante de lo que hubo, en una de las colecciones españolas más importantes de estas caracteristicas. Analizada en su conjunto, permite observar su continuidad, reflejada en la presencia de instrumentos representativos de todas las épocas mencionadas, así como de las más variadas disciplinas científicas y técnicas del siglo XIX.

\section{Introducción. El origen de una colección}

La primera gran adquisición de instrumentos con destino al Colegio Imperial se llevó a cabo en Inglaterra en torno a 1750. Este primer grupo, al que pudo sumarse algún instrumento realizado en España o trasladado desde otra institución, estaba destinado fundamentalmente a las disciplinas de Matemáticas (en sus ramas más prácticas, como Dibujo, Perspectiva o Topografía) y Astronomía, y muy especialmente al observatorio astronómico que Juan Wendlingen planeaba instalar en el Colegio (Guijarro, 2002, 104).

En 1767 los jesuitas fueron expulsados de España y el centro pasó a depender de la Corona, convirtiéndose en los Reales Estudios de San Isidro. Con la intención de convertir a esta institución en pionera dentro de la reforma educativa ilustrada, se realizó una segunda compra importante de material para modernizar el instrumental de Física experimental, principal disciplina que, desde entonces y hasta casi un siglo después, requería el uso de instrumentos científicos. En este caso los instrumentos no se adquirieron fuera, sino que su construcción estuvo a cargo del fabricante español Diego Rostriaga (Guijarro, 2002, 122).

Tras esta nueva adquisición ya no se realizaría ninguna compra importante hasta mediados del siglo siguiente, aunque sí la adquisición puntual de nuevas piezas. En inventarios que se conservan de $1774,1775-77$ y $1777-1814$ (Guijarro, 2002, 189-194) se hace referencia a un total de 108 instrumentos, que son los que a principios del siglo XIX debian constituir la colección.

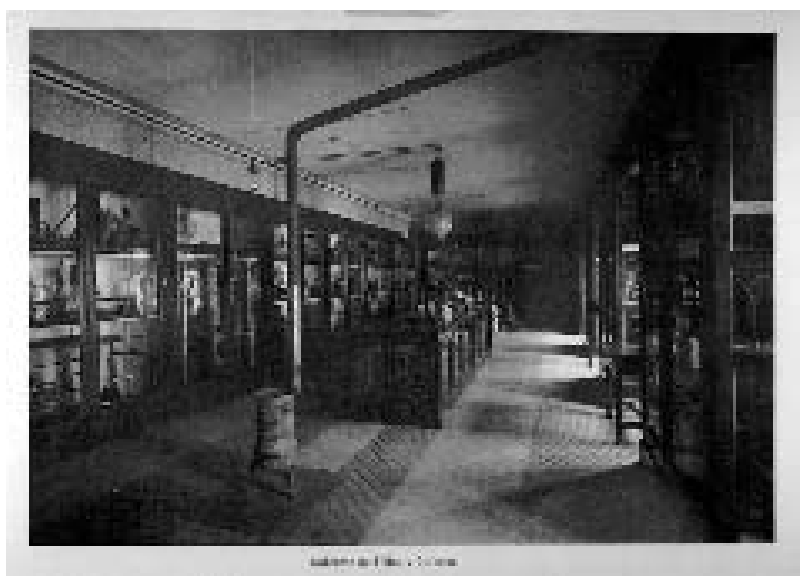

Figura 1. Gabinete de Física y Química del Instituto San Isidro.

En medio de numerosos vaivenes políticos que repercutieron en la estabilidad de la instrucción pública española, en 1823, con la vuelta de Fernando VII al poder, el centro fue restituido a la Compañia de Jesús, hasta que ésta fue definitivamente expulsada en $1836^{4}$. En un inventario que se conserva de ese año, realizado por el profesor Mariano Santisteban, figuran 210 instrumentos, de los cuales 84 todavía pueden contemplarse en el MUNCYT (Guijarro, 2002, 189-194).

\section{Política educativa y adquisición de instrumentos en el siglo XIX}

Después de esa fecha se inició una nueva etapa en la que España se incorporó, tardíamente tras el colapso en el desarrollo de la ciencia provocado por la guerra de la Independencia, al movimiento científico que estaba teniendo lugar en el resto de Europa. Esta nueva etapa, 
ya más estable en materia de enseñanza y caracterizada por una tendencia al centralismo, se inició especialmente con la gran reforma que supuso en 1845 el conocido como plan Pidal, que significará un nuevo plan de estudios para las enseñanzas secundaria y superior. Su promotor, Antonio Gil de Zárate, en su esperanzadora circular de 1846 (Gil de Zárate, 1846, 545-557), comunicaba a los presidentes de las Juntas inspectoras de los institutos de segunda enseñanza el deseo de abastecerlos con los instrumentos precisos para el aprendizaje de las ciencias físicas y naturales, instaba a los profesores a utilizarlos no sólo en la enseñanza, sino en sus propias investigaciones e incluía dos listas de los aparatos que debian componer un gabinete de física y un laboratorio de química. Estas listas constituian de hecho una referencia de lo que se adquiriría para cumplir tales objetivos, pues en ellos figuraban los precios correspondientes a los catálogos de los fabricantes franceses seleccionados para suministrar el instrumental (Lerebours, Deleuil y Pixii para los gabinetes de Física, y Lizé, Clech y los hermanos Rousseau para los gabinetes de Química), así como una nota de los útiles de química que podian adquirirse en España.

Tras este primer paso, en 1857, con la Ley Moyano (BOMF, 1857a, 5-26 y 1857b, 49-67), se produjeron los cambios más significativos en el instituto San Isidro, ya que pasó a integrarse en las enseñanzas medias. Bajo una concepción utilitaria de la ciencia, que buscaba inducir con su promoción una mejora de la industria española, los estudios de esta disciplina, que hasta entonces tenían un carácter propedéutico y estaban incluidos en la Facultad de Filosofía y Letras, pasaron a configurar la Facultad de Ciencias Exactas, Físicas y Naturales. Además, con esta nueva Ley se prestaba, al menos teóricamente, más atención a la enseñanza práctica de las ciencias: en el bachillerato en Ciencias figuraban, entre otras asignaturas (Colección, 1860, 214-215), Ampliación de Física experimental y Química general $y_{1}$ como veremos, los centros de enseñanza secundaria pasaron a ofrecer no sólo formación general sino también profesional.

A pesar de las buenas intenciones, la realidad sería que sucesivos decretos, que seguirian apareciendo en 1880, recordaban sin éxito la obligatoriedad de las asignaturas prácticas, por otra parte difíciles de impartir sin unos gabinetes capaces de cubrir las necesidades de docentes y alumnos, tal y como se refleja en el siguiente texto, correspondiente a la memoria del curso 1860-61 del San Isidro (Memoria, 1860, 12-13):

La índole de la enseñanza que en él se cursan, la escasa edad de la mayoría de los alumnos que las estudian, exigen que, si se han de obtener buenos resultados de la instrucción, sean esencialmente prácticas, y esto es imposible al no contar con buenos y abundantes medios de demostración. Hacer teórica una asignatura que debe ser experimental; obligar al alumno a que recuerde solo palabras y no exprese ideas, es infundir el desaliento, es amortiguar o entorpecer en su germen inteligencias privilegiadas, es recargar la memoria de nombres inútiles y fomentar una erudición superficial, origen del charlatanismo y de grandes males, cuyos frutos se recogen luego, ya abandonando el alumno los estudios clásicos, fundamentos de toda educación literaria o científica, ya cursando $\sin \mathrm{fe}$, sin emulación, y solo por la esperanza de lucro, las importantes asignaturas de los estudios superiores.

Estas asignaturas buscaban fundamentalmente la reproducción de algunas experiencias clásicas de la física e incluso la demostración de descubrimientos recientes, no sólo científicos sino también tecnológicos, que se incluian dentro de los programas como aplicaciones de los conocimientos teóricos. Esta concepción se refleja, como veremos más adelante, en la presencia de instrumentos técnicos tanto en los manuales como en las colecciones que se conservan.

Con posterioridad a la Ley Moyano se realizarian sucesivas compras que figuran reflejadas de forma muy general, con referencias concretas sólo a aparatos de cierta importancia, en las memorias que el instituto publicó cada año desde 1858. Así, en un inventario realizado por Mariano Santisteban en 1878 se hace referencia a la adquisición, entre 1835 y ese mismo año, de 341 aparatos de Física y 74 de Química (Santisteban, 1875, 191-206). No obstante, y a pesar de la presencia de nuevos instrumentos, no se alcanzó una situación más o menos regularizada, en lo que a la política de compras de material científico se refiere, hasta el año 1911, fecha en la que se creó el Instituto de Material Científico (Romero, 1998), destinado a unificar las compras de este tipo de material destinadas a centros estatales docentes y de investigación.

ARBOR Vol. 187749 mayo-junio [2011] 561-571 ISSN: 0210-1963 


\section{Programas, manuales y gabinetes}

Como avanzamos, una parte significativa de la tecnología, concebida como "ciencia aplicada", se presentaba en los libros de Física y Química ligada a los contenidos teóricos. Esta particular concepción de las relaciones entre la ciencia y la tecnología se trasladó a la práctica educativa; así, desde la Ley Moyano, en los institutos se cursaban tanto los estudios generales como los que capacitaban para las profesiones industriales. Como consecuencia, los manuales que reflejaban tanto las disposiciones legales como esta mentalidad podían emplearse en los institutos y en las escuelas de artes y oficios, como ocurría con los textos de Eduardo Lozano y Ponce de León, Elementos de Física y Elementos de Química (este en particular tenía un apéndice dedicado a industrias químicas) (López, 1999, 117 y 199). Si observamos el programa de Física y Química seguido en el año 1884 en el instituto San Isidro de Madrid, comprobaremos que, por ejemplo, en el apartado dedicado a la "electricidad dinámica" se contemplaba un número significativo de dispositivos tecnológicos comunes en el siglo XIX, como los diferentes modelos de pila, los telégrafos y los aparatos de inducción eléctrica, entre los que se incluia el teléfono. Sin duda, los intereses descritos influyeron en el tipo de material que se manejaba o adquiría en los centros de segunda enseñanza. Correspondiendo a este enfoque encontramos, entre los instrumentos que guarda el MUNCYT procedentes del instituto San Isidro, objetos tales como diversos tipos de pilas o variados modelos de telégrafo impresor.

Esta concepción de las relaciones entre los contenidos científicos y los técnicos es por tanto lo que explica el interés que en el siglo XIX tenía este centro por adquirir las últimas novedades tecnológicas. El reflejo de este interés se observa en la presencia en dicha colección del ejemplar n. ${ }^{\circ} 21$ del primer modelo de fonógrafo comercializado por Edison, el tin-foil ${ }^{5}$, o de objetos tales como cámaras fotográficas estereoscópicas y de microfotografía o accesorios de revelado de daguerrotipos.

En la Ley de 1 de agosto de 1876 (Gaceta de Madrid núm. 216, de 3 de agosto de 1876) se estableció como obligatoria la enseñanza de la Agricultura en el Bachillerato, hecho que impulsó, además, la creación de gabinetes específicos para la disciplina, como el que ilustra la fotografía, perteneciente al instituto San Isidro. La disposición de estos instrumentos confería a los centros una ocupación que proporcionaba ingresos adicionales: en los establecimientos se llevaban a cabo análisis y ensayos de tierras, aguas y otros líquidos, que solicitaban los agricultores de acuerdo con las tarifas oficiales aprobadas (Damián, 1999, 210). Como testimonio de estas actividades podemos señalar una de las piezas recuperadas este curso, un hidrotímetro del siglo XX (n. inventario S.I./2008/11/13).

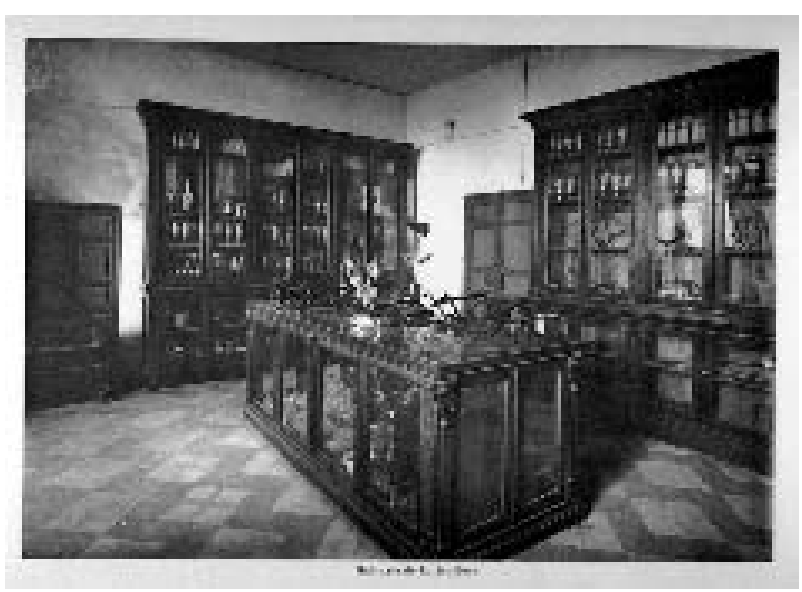

Figura 2. Gabinete de Agricultura del instituto San Isidro.

Para una aproximación al lugar ocupado en la práctica educativa por los materiales, sustancias y costosos aparatos adquiridos hay que dirigir la mirada a los manuales que se empleaban en la enseñanza. En la segunda mitad del siglo XIX, los autores españoles fueron sustituyendo paulatinamente a los franceses, dominantes en las obras manejadas en nuestro país hasta ese momento (López, 1999, 108). Así, se comenzaron a emplear, entre otras, los textos de Venancio González Valledor y Juan Chávarri (1848), Programa de un curso elemental de Física y nociones de Química; Manuel Rico y Mariano Santisteban (1869), Manual de Física y Elementos de Química, Madrid; Bartolomé Feliú (1883), Curso elemental de Física experimental y aplicada y Nociones de Química inorgánica, Barcelona. Estos manuales participaban de algunos rasgos comunes:

a) Contenidos muy similares. Todos respondian a un esquema ya establecido en la Ilustración, que comenzaba con la presentación de las propiedades más generales de los cuerpos y seguía con las cuestiones de mecánica, propiedades del aire, el calor, la luz, la 
electricidad, el magnetismo, terminando algunos con consideraciones relativas a la meteorología.

b) Excesivo volumen y dificultad de una parte significativa de sus contenidos, inaccesibles en muchos casos a los alumnos de la enseñanza secundaria, a quienes en principio estaban destinados.

c) Descripción de experiencias y máquinas para que fueran empleadas en las clases exclusivamente por el profesor como complemento de sus explicaciones.

Sólo a principios del siglo XX se introdujeron en los textos algunos planteamientos pedagógicos que rivalizaban con los tratamientos enciclopédicos de la centuria anterior. Estos cambios coincidieron con ideas renovadoras en la educación secundaria promovidas por la Institución Libre de Enseñanza y la Junta para la Ampliación de Estudios, que alcanzaron una difusión relativa por medio del InstitutoEscuela. Los nuevos presupuestos pedagógicos afectaron igualmente a la concepción y finalidad de los gabinetes. Se huía de una metodología basada en las demostraciones con "aparatos costosos, raros y desconocidos para los alumnos y se apostó por la construcción de materiales por parte de los alumnos o por la utilización de materiales sencillos" (López Martínez, 1999, 240), como ilustra la obra de Estalella (7.a edición de 1973), Ciencia recreativa, Barcelona, Gustavo Gili, y J. Estalella y J. Kleiber (1914), Compendio de Física y Química, Barcelona, Gustavo Gili.

\section{Los proveedores de instrumentos. Avatares de una colección}

Si analizamos los instrumentos firmados que constituyen la colección del instituto San Isidro (considerando tanto los que quedan en el centro como los que se conservan en el MUNCYT), observaremos que una gran parte de las piezas han sido adquiridas en el extranjero, variando su origen en función de las épocas. Así como en el siglo XVIII, como vimos, los instrumentos proceden de Inglaterra o de los talleres de Diego y Celedonio Rostriaga, durante gran parte del XIX destaca la presencia de Francia. En este último siglo, no obstante, debemos hablar de dos etapas bien diferenciadas: hasta la década de 1870 domina Francia y, aunque existe algún fabricante español, como Grasselli, se aprecia la escasez de una industria nacional consolidada, situación que empieza a cambiar en el último tercio del siglo (Guijarro, 1999, 9); por otra parte, a finales del XIX y en la primera mitad del XX se aprecia una mayor diversidad de paises productores, aunque encabezados por Alemania. Así, en este último período, podemos encontrar instrumentos procedentes de talleres ingleses (como Casella o Griffin), alemanes (como Leybold, F. Ernecke o Max Kohl) o franceses (como Ducretet o Koenig); finalmente, a medida que avanza el siglo XX, comienza a apreciarse en las colecciones la presencia de productores españoles como Aramburo, Torrecilla, Sogeresa (fundada en 1918), Cultura (fundada en 1924), o ENOSA (fundada en 1951).

Por otra parte, si bien los ejemplares que constituyen el gabinete de Historia Natural han permanecido en el centro desde sus orígenes hasta nuestros días, no sucede lo mismo con las piezas de los gabinetes de Física y Química del actual instituto San Isidro.

En primer lugar, desde que en 1845 se creó la Facultad de Ciencias de la Universidad Central y hasta que en 1855 se terminaron las obras del nuevo edificio de Noviciado, que se compartiría con el actual instituto Cardenal Cisneros (e incluso unos años después), al no disponer la Facultad de un edificio propio, las cátedras de Física y Química se impartían en el ya instituto San Isidro. Esta situación provocó que durante este periodo y hasta que la situación no se aclaró en 1850 (González y Martín, 2000, 34), no se supiese con certeza qué instrumentos constituían cada gabinete pues, a pesar de tratarse de niveles diferentes, entonces el material era prácticamente el mismo en institutos e universidades.

En segundo lugar, tras la fundación en Madrid en 1837 de la Facultad de Filosofía (con estudios superiores de Física), sus profesores empezaron a solicitar material para las asignaturas experimentales. Dada la falta de fondos para cubrir estas necesidades, y aprovechando el control que la Universidad Central ejercía sobre los otros centros educativos de nivel inferior, se recurrió a tomarlos prestados de otras instituciones. Entre ellas figuraron los Reales Estudios de San Isidro, a cuyo director se le solicitó que prestase algunos de sus instrumentos para abastecer al catedrático de Fisica de la Universidad. A pesar de su oposición, y tras intentar otras alternativas, en 1857 se trasladaron 19 instrumentos (Santisteban, 1875, 52-54 y 130-131), que no fueron devueltos hasta 1872 (Santisteban, 1875, 108).

Finalmente, ya en el siglo XX, la mayoría de los instrumentos de física y química que se conservaban se depositó en el . 
MUNCYT, que pasó a ser propietario de ellos unas décadas después. No obstante, y gracias al programa Ciencia y educación en los institutos madrileños de educación secundaria a través de su patrimonio cultural, 1837-1936 (CEIMES) se ha podido iniciar el estudio del material que quedó entonces en el actual instituto San Isidro y que ha desvelado ser más cuantioso e interesante de lo que en principio parecía.

\section{ReCuPERACIÓN del MATERIAL CIENTífico Y TECNOLÓGICO}

Durante el curso 2008-2009 se han realizado tareas destinadas a la recuperación de los instrumentos científicos antiguos que aún se conservan en el laboratorio de física del instituto San Isidro. En total y hasta el momento, dado que el trabajo tendrá continuidad en los cursos siguientes, se han restaurado 40 piezas, en su gran mayoría pertenecientes al siglo XIX, y en menor proporción a las primeras décadas del XX. Para la realización de este trabajo se han consultado tanto manuales de texto de la época como publicaciones, nacionales y extranjeras, relacionadas con el estudio histórico de instrumental científico existente en museos y centros educativos. Entre los primeros destacamos textos como los de Feliú o Ganot y entre las segundas, obras como, además de las ya citadas, Nineteenth-Century Scientific Instruments (Turner, 1983), Instruments of science (Bud y Warner, 1998) o Abriendo las cajas negras (Bertomeu y García, 2002).

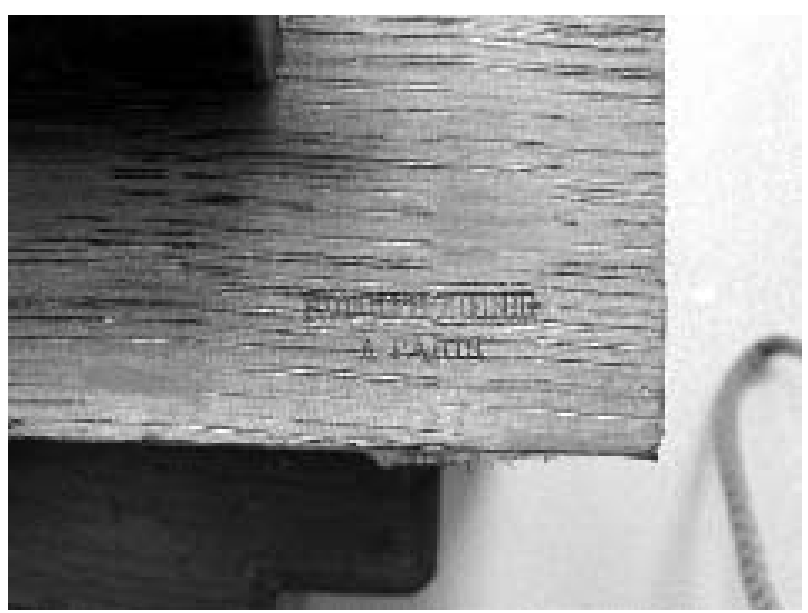

\section{Metodología}

Las tareas de recuperación han consistido en:

- Localización de instrumentos dispersos situados en el laboratorio, el departamento y el almacén de física y reubicación en una zona adecuada.

- Identificación de los mismos, asignándoles un nombre y número de inventario que figuran en una tarjeta de cartulina que cuelga de cada instrumento (este último figurará también siglado en la pieza). El número de inventario consta de las iniciales del centro (S.I.) seguidas de tres grupos de números que hacen referencia, respectivamente, al año en que se inició el inventario (2008), al número que ocupa la pieza en la colección (hasta ahora del 1 al 40 ) y a la sección a que pertenece la pieza de acuerdo a las siguientes referencias: 1. Acústica, 2. Calor, 3. Electricidad, 4. Electromagnetismo, 5. Física Atómica, 6. Magnetismo, 7. Mecánica, 8. Mecánica de fluidos, 9. Meteorología, 10. Metrología, 11. Óptica, 12. Accesorios, 13. Ciencias Químicas.

- Realización de varias fotografías de cada objeto, tanto de este en su conjunto como de detalles que puedan ser de interés para aportar más información sobre el instrumento, como la firma del fabricante, escalas, la placa del distribuidor o etiquetas del profesor que lo utilizaba en sus tareas docentes.

- Inventariado y precatalogación

- Datación. Aunque dadas las características de esta publicación no podemos extendernos en este punto, al menos

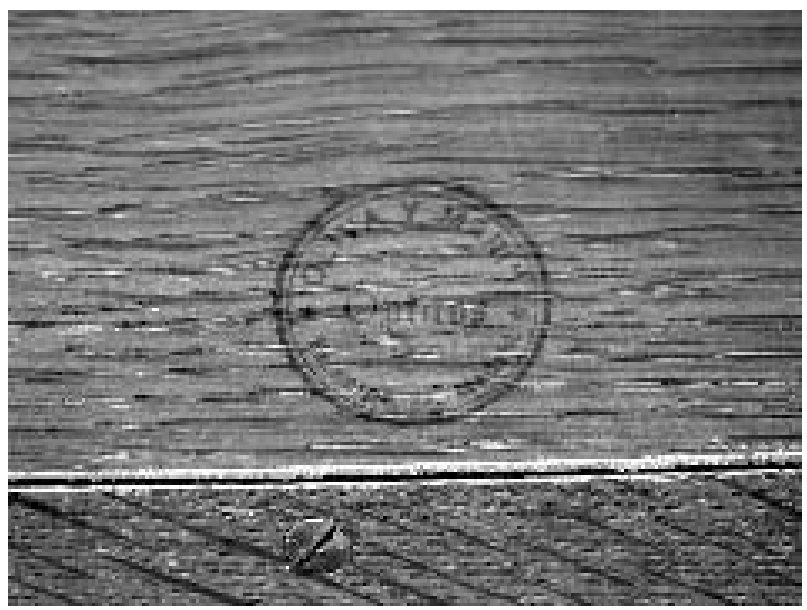

Figura 3. Fotografías de detalles: la firma del fabricante (Rudolph Koenig) y el sello del distribuidor (Oliva y Marín) del "pequeño regulador de viento" (SI/2008/36/1). 
mencionaremos algunos aspectos, pues esta es una de las tareas más complejas en la mayoría de las ocasiones. No es este el caso de instrumentos de los que se conoce el año de compra por haber quedado registrado en inventarios, memorias u otro tipo de documento, o de aquellos que llevan la firma del fabricante y se dispone de su catálog $0^{6}$ o se conocen los períodos en que trabajó. En el resto de los casos nos hemos fijado en todo aquello que puede aportar información. En unas ocasiones se ha tenido en cuenta la fecha atribuida a instrumentos de similares características en catálogos de otras colecciones; en otras hemos prestado atención al tipo de letra o el estilo de etiquetas o adornos, común a otras manifestaciones artísticas de la época; finalmente, también nos han dado pistas las técnicas de fabricación (por ejemplo,

DEPARTAMENTO: Disciplina a que pertenece (Física, Industria, Matemáticas, Metrología, Química).

SECCIÓN: Ramas en que se dividia la disciplina correspondiente en la época a que pertenece el instrumento.

N. ${ }^{\circ}$ INVENTARIO

NOMBRE

UBICACIÓN

DIMENSIONES

MATERIAS

TRANSCRIPCIÓN: Inscripciones que Ileve la pieza en etiquetas, escalas, placas de fabricantes o distribuidores, etc.

DATACIÓN: Período aproximado, en años, durante el que se construyó la pieza.

CONSTRUCTOR/PRODUCTOR: Persona o empresa que lo construyó.

AÑO: Año de fabricación.

DISTRIBUIDOR: Empresa encargada de hacer llegar la pieza al consumidor, sirviendo de intermediario entre éste y el fabricante.

LUGAR DE PROCEDENCIA: Lugar de fabricación.

DESCRIPCIÓN TÉCNICA: Descripción del funcionamiento del instrumento y la finalidad a que estaba destinado dentro de su contexto histórico.

CONSERVACIÓN: Estado de conservación de la pieza.

OBSERVACIONES: Cualquier información que desee hacerse constar y no pueda incluirse en ninguno de los campos mencionados.

BIBLIOGRAFÍA: Obras consultadas (manuales de la época, catálogos de fabricantes o de instituciones con instrumentos similares, publicaciones de los trabajos originales de los inventores, obras especializadas, etc.), páginas web, memorias, inventarios y documentación que acompaña a la pieza.

AUTOR Y FECHA DE CATALOGACIÓN en los tornillos del XIX se aprecia una irregularidad debida a su fabricación artesanal que no se da en los del XX) o los materiales con los que está construido (en general, por ejemplo, el latón y las maderas nobles son propias del XIX, el metal niquelado y las maderas teñidas más propias de principios del XX, el cromado y los plásticos de mediados y finales del XX). En una de las piezas que se documenta al final de este artículo se adjunta un cuadro en el que se reflejan estos últimos aspectos aplicados a la datación de lámparas de incandescencia fabricadas por Edison.

Toda la información relativa a la documentación de la pieza ha sido recogida en una base de datos con los siguientes campos:

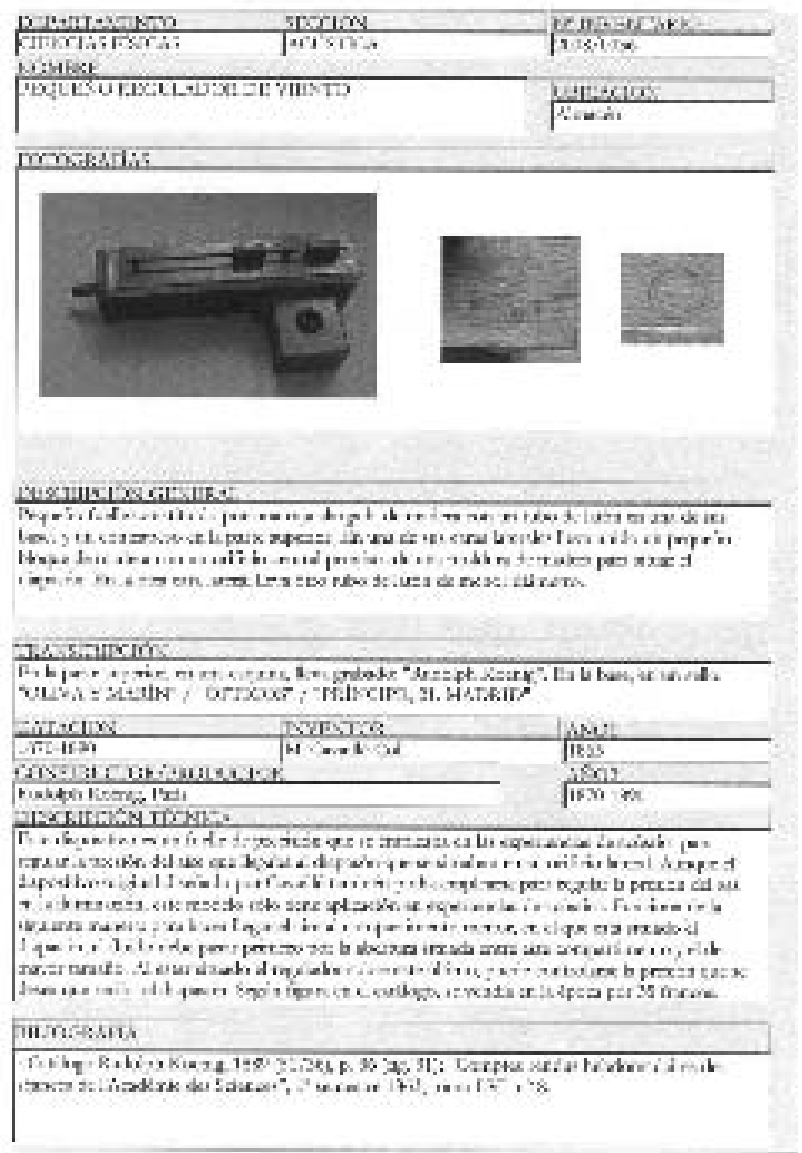

Figura 4. Ejemplo de ficha de catalogación.

ARBOR Vol. 187749 mayo-junio [2011] 561-571 ISSN: 0210-1963 


\section{Dos instrumentos representativos}

Para dar una idea de las características de la colección y del trabajo realizado, hemos seleccionado dos piezas muy diferentes entre sí en muchos aspectos que por sus características nos permiten tener una idea del tipo de instrumento que se adquiría para la enseñanza en el s. XIX en el instituto San Isidro. Se trata de un pequeño regulador de viento y de una lámpara de filamento incandescente.

\section{Pequeño regulador de viento}

El dispositivo que observamos en la fotografía es un fuelle de precisión que se empleaba en experiencias de acústica para regular la presión del aire que llegaba al instrumento situado en su orificio lateral, generalmente una sirena.
Aunque el diseño original ideado por Cavaillé-Coll también podía emplearse para regular la salida del gas de iluminación, sabemos que este modelo estaba destinado a experiencias de acústica porque su fabricante, Rudolph Koenig, estaba especializado en esta disciplina de la física ${ }^{7}$, y con tal aplicación figura en su catálogo (Koenig, 1889, 36) con un precio de 35 francos.

Construido en torno a 1880, consta de dos compartimentos unidos por una abertura en la que está situada una válvula lenticular. El aire introducido por el tubo de entrada pasa por la válvula y llega a la sirena (o al instrumento que dispongamos en el tubo de salida) con una presión constante, presión que puede modificarse de forma continua deslizando el contrapeso situado en la parte superior.
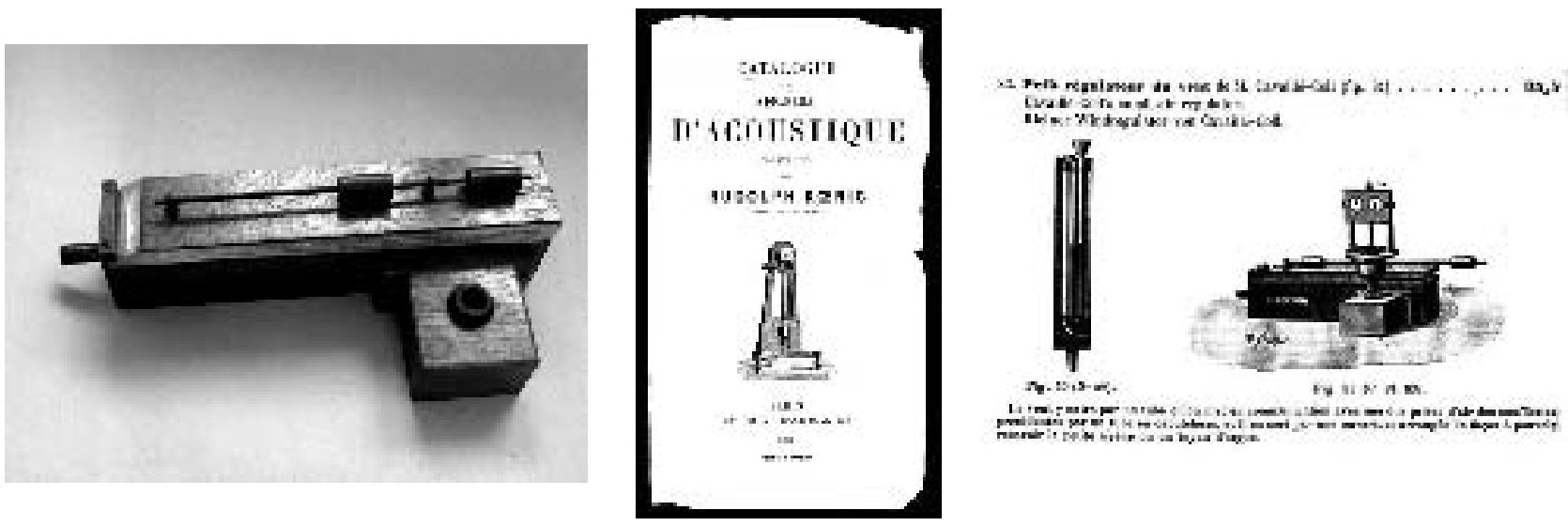

Figura 5. Pequeño regulador de viento, catálogo de Rudolph Koenig y detalle de la página del catálogo donde figura el regulador.

Si bien la relación entre tono y frecuencia ya se conocía desde principios del siglo XVII, no es hasta el XIX cuando se realizan mediciones precisas de esta relación, mediciones que este pequeño fuelle regulador está destinado a facilitar. Fue presentado por su inventor ante la Royale Académie des Sciences de París en 1863 y recogida dicha presentación en las actas de las sesiones de dicha institución (Cavaillé-Coll, 1863, 339).

Surgido de experiencias destinadas a regular la presión del aire en fuelles de grandes órganos, se proponía tam- bién como una herramienta útil en experiencias que requiriesen precisión a la hora de obtener un flujo constante de aire $u$ otro gas, como, por ejemplo, en laboratorios de Química, para obtener una temperatura constante en calefacción por gas o en gabinetes de Física, para estudiar leyes de fluidos aeriformes. No obstante, su autor destacaba como una de sus aplicaciones más interesantes la que precisamente tiene este modelo, regular el giro de la sirena ideada por Cagniard de Latour": "Admirable instrumento [...] que hasta el momento no ha podido emplearse en experiencias precisas debido a la dificultad 
que supone regular la velocidad de su movimiento". Para ilustrar sus aplicaciones, describía a continuación una serie de experimentos realizados con el regulador en sus dos versiones, la angular, presentada aquí, y la horizontal, con aplicación en tubos de órgano, concluyendo con una tabla en la que se reflejaban las frecuencias de diversos tubos de órgano afinados, según el nuevo diapasón normal (870 vps).

\section{Lámpara de filamento incandescente}

Las lámparas eléctricas de filamento incandescente surgieron en la década de 1880 con la aparición de la luz eléctrica y como sistema alternativo a la iluminación por combustión de gas. Se basan en el principio de la transformación de la energía eléctrica en calor. Para ello se hace pasar una corriente continua o alterna por un conductor encerrado en una ampolla al vacío y lo suficientemente fino, rígido y resistente eléctricamente como para ponerse incandescente sin fundirse ni deformarse.

Este modelo, en concreto, correspondiente a la patente de Edison, fue fabricado en torno a 1885. Aunque en la época diversos inventores idearon otros tantos modelos de lámpara incandescente, fue la patente de filamento de carbón concedida a Edison en 1880 la que aventajó a las demás. Los motivos de este mayor éxito residieron fundamentalmente en que Edison supo identificar y desarrollar los diferentes aspectos que condicionaban la viabilidad de este invento (Friedel, 1987, 121-122), como la idoneidad del material incandescente, el alto vacío o un sistema eficaz de suministro eléctrico.

En los gabinetes de Física, en general, este tipo de aparato se empleaba fundamentalmente para indicar el paso de la corriente en diversas experiencias de electricidad. No obstante, la presencia en la colección del instituto San Isidro de este modelo, uno de los primeros patentados por Edison, también puede deberse a las razones antes comentadas de incluir en las enseñanzas de Física, como aplicaciones de contenidos teóricos, las últimas innovaciones tecnológicas.

Para finalizar, y como ejemplo de los aspectos antes mencionados relativos a la datación de las piezas, recogemos en un cuadro en el que la observación de determinadas características específicas de la base de una lámpara nos permiten asignarle una fecha aproximada, información que se ha empleado para datar el modelo que aquí presentamos.
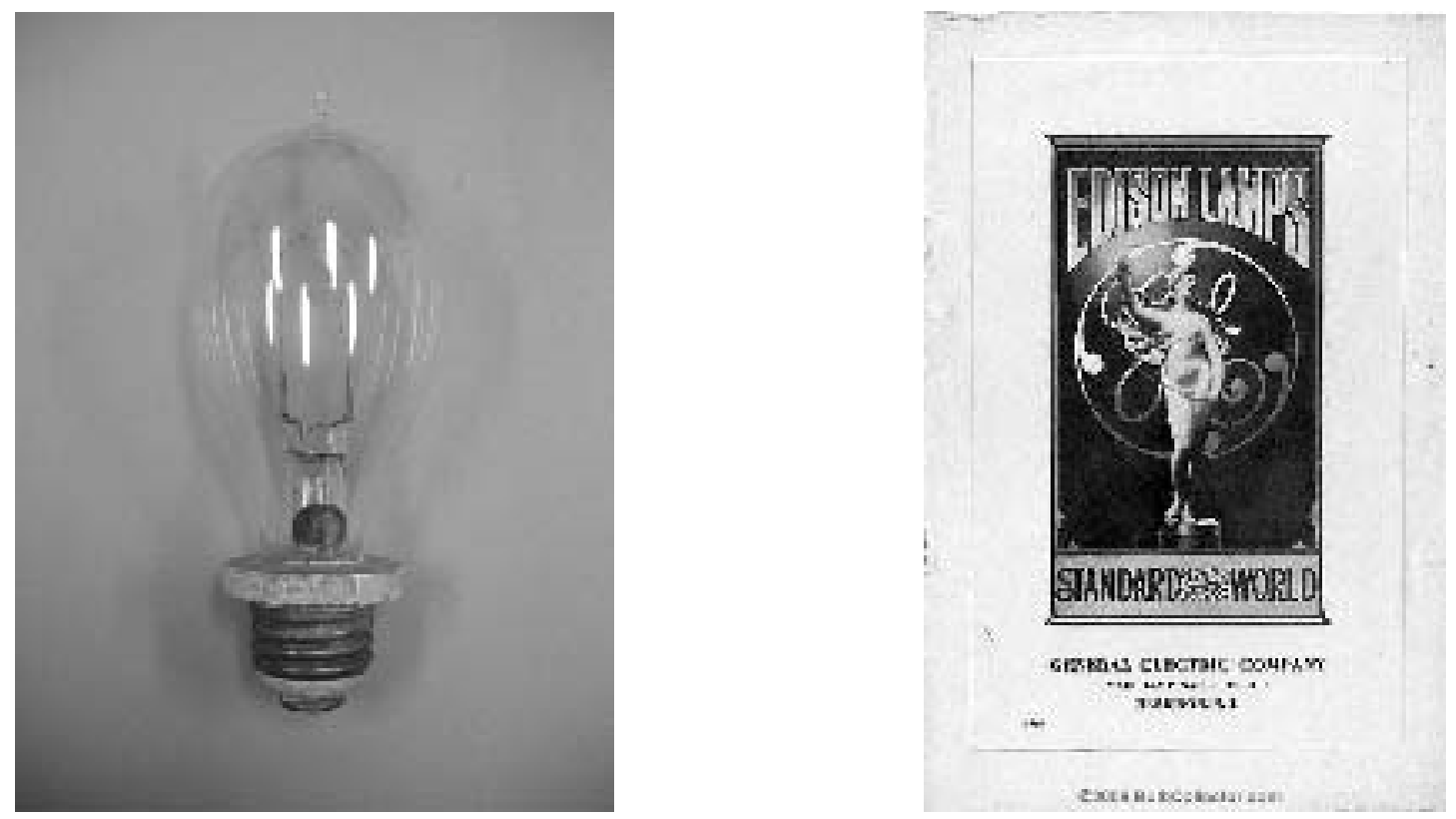

Figura 6. Lámpara de filamento incandescente de Edison y cubierta de un catálogo de este fabricante (1902). 
CRONOLOGÍA DE LOS MODELOS DE LÁMPARA DE EDISON SEGÚN LAS CARACTERÍSTICAS DE SU BASE

\begin{tabular}{|l|l|}
\hline $1879-1880$ & Base de madera con terminales de contacto. \\
\hline 1880 & Terminales en forma de láminas de cobre. \\
\hline 1880 & Base con rosca de madera \\
\hline 1881 & Base de madera con pequeña rosca de latón y anillo. \\
\hline 1881 & Base de escayola con pequeña rosca de latón y anillo. \\
\hline $1881-1884$ & Base de escayola con pequeña rosca de latón, anillo y botón de contacto en el extremo inferior. \\
\hline $1884-1888$ & Base de escayola con pequeña rosca de latón y botón de contacto en el extremo inferior (sin anillo). \\
\hline $1888-1897$ & Aislante de escayola y cemento con base provista de larga rosca y botón de contacto. \\
\hline $1896-1901$ & Base porcelana y latón según la forma moderna. \\
\hline $1901-$ & Base según la forma moderna con aislante de vidrio. \\
\hline
\end{tabular}

El presente trabajo nos ha permitido profundizar en el análisis de una colección de gran interés, y supone un primer paso que aporta valiosa información para posteriormente estudiar cómo era la enseñanza de las disciplinas científi- cas en el siglo XIX y el lugar ocupado por los instrumentos en estos planteamientos educativos. Está previsto que tenga continuidad en los próximos cursos, hasta completar la catalogación del material existente.

\section{NOTAS}

1 Quiero agradecer a la directora del centro, Isabel Piñar Gallardo, y al jefe de estudios, Rafael Martín Villa, su colaboración en las tareas de recuperación del material antiguo.

2 Por otra parte, y dentro del programa CEIMES, Eduardo Fernández ha llevado a cabo el estudio del material perteneciente al gabinete de Historia Natural.

3 Para la Academia de Matemáticas, véase Vicente y Esteban (1991, 98-109).

4 Para la historia general del instituto véase Simón, 1959; para el período de 1845 a 1936 véase Navarro, 1991.

5 Número de inventario 85/4/597.

6 Pueden consultarse numerosos catálogos de fabricantes en la página web de la Smithsonian Institution: $<$ http://www.sil.si.edu/digitalcollections/trade-literature/scientificinstruments/CF/SIcompany-namesdrilldown.cfm>.

7 Para más información sobre este interesante fabricante que con un pequeño taller abasteció a gabinetes de todo el mundo (por ejemplo pueden encontrarse numerosos instrumentos suyos en colecciones de Canadá, Coimbra o Florencia) veáse P. Brenni (1995).

8 Instrumento ideado en 1819 para producir un sonido a partir de una corriente de aire y determinar su frecuencia. En el MNCYT existe un ejemplar procedente del instituto San Isidro con el n. ${ }^{\circ}$ de inventario 85/4/577.
Recibido: 1 de marzo de 2010

Aceptado: 30 de junio de 2010 


\section{BIBLIOGRAFÍA}

Bertomeu Sánchez, José Ramón y García Belmar, Antonio (ed.) (2002): Abriendo las cajas negras, Valencia, Universidad de Valencia.

Boletín Oficial del Ministerio de Fomento (1857, 1 y 8 de octubre), tomo XXIV, año VI, n. 301 (a) y n. ${ }^{\circ} 302$ (b).

Brenni, P. (1995): "19 ${ }^{\text {th }}$ Century French Scientific Instrument Makers: VI: The Triumph of Experimental Acoustics: Albert Marloye (1795-1874) and Rudolph Koenig (1832-1901)", Bulletin of Scientific Instrument Society, 44, 13-17.

Bud, R. y Warner, D. J. (eds.) (1998): Instruments of science. An Historical Encyclopedia. Garland Encyclopedias in the History of Science. Vol. 2, Nueva York y Londres, Science Museum, Smithsonian Institution, Garland Publishing.

Cavaillé-Coll, A. (1863, 1. ${ }^{\circ}$ semestre): "Sur une nouvelle soufflerie de précision, munie d'un nouveau système de régulateurs de la pression de l'air et des gaz, et sur quelques applications de cet appareil à des expériences d'acoustique et à la régularisation de l'émission du gaz d'éclairage", Comptes rendus hebdomadaires des séances de l'Académie des Sciences, LVI, 8, pp. 314 y 339.
Colección Legislativa de España (1. ${ }^{\circ}$ semestre de 1860), tomo 83, Madrid.

Friedel, Robert e Israel, Paul (1987): La luz eléctrica de Edison, Barcelona, Bellaterra.

Ganot, A. (1909): Tratado Elemental de Fisica, Madrid.

Gil de Zárate, Antonio (15 de octubre de 1846): Boletín Oficial de Instrucción Pública, tomo IX, segunda serie, año 6. ${ }^{\circ}$, n. 19.

González de la Lastra, Leonor y Martín Latorre, Rosa M. ${ }^{a}$ (2000): Instrumentos científicos para la enseñanza de la Física, Madrid: Ministerio de Educación y Cultura.

Guijarro Mora, Victor (2002): Los instrumentos de la ciencia ilustrada. Física experimental en los Reales Estudios de San Isidro de Madrid (1770-1835), Madrid, UNED.

Guijarro Mora, Victor (1999): "Procurance \&t Manufacture of scientific instruments in Spain during the 18th. and 19th. Centuries", Scientific Instrument Society Bulletin, n. ${ }^{\circ}$ 62, 7-10.

Koenig, Rudolph (1889): Catalogue des Appareils d'Acooustique construits par Rudolph Koenig, París (51736).

López Martínez, José Damián (1999): La enseñanza de la Física y Química en la educación secundaria en el primer ter- cio del siglo XX en España, tesis leída en la Universidad de Murcia, Murcia, 1999.

Memoria acerca del estado del Instituto de $1 .^{a}$ clase de San Isidro, incorporado a la Universidad Central (1860), Madrid.

Navarro Jurado, A. (1991): Historia del Instituto de Segunda Enseñanza de San Isidro, 1845-1936, tesis doctoral, Madrid, Universidad Complutense.

Romero, A. (1998): "Dos políticas de instrumental científico: el Instituto del Material Científico y el Torres Quevedo", Arbor, 160, n. ${ }^{\text {s }}$ 631-632 (julioagosto), 359-386.

Santisteban, M. (1875): Breve historia de los gabinetes de física y química del Instituto de San Isidro de Madrid, Madrid.

Simón Díaz, José (1959): Historia del Colegio Imperial de Madrid, Madrid, CSIC, Instituto de Estudios Madrileños, 2 Tomos.

Turner, G. L'E. (1983): Nineteenth-Century Scientific Instruments, Londres, Sotheby's Publications.

Vicente Maroto, M. I. y Esteban Piñeiro M. (1991): Aspectos de la Ciencia aplicada en la España del siglo de oro, Salamanca, Junta de Castilla y León, Consejería de Cultura y Bienestar Social. 